\title{
Fogitare
}

ARTIGO ORIGINAL

\section{NECESSIDADES DOS IDOSOS NA AUTOGESTÃO DA DOENÇA CRÔNICA: PERSPECTIVANDO UM PROGRAMA DE INTERVENÇÃO DE ENFERMAGEM}

\author{
THE ELDERLY NEEDS IN SELF-MANAGEMENT OF CHRONIC \\ DISEASE: ENVISIONING A NURSING INTERVENTION PROGRAMME
}

\author{
Celeste Bastos ${ }^{1}$ (1) \\ Célia Santos ${ }^{1}$ (i) \\ Maria Manuela Martins ${ }^{1}$ (1) \\ Carla Fernandes ${ }^{1}$ (1) \\ Lígia Lima ${ }^{1}$ (i)
}

\begin{abstract}
Objective: To characterise the health profile of community-dwelling older people and identify the domains of the disease self-management process for nursing interventions. Method: Mixedmethod study, with 48 participants from Porto, Portugal, between September 2018 and July 2019. A questionnaire was used to assess disease and health-related variables, and a semi-structured interview was also undertaken focused on chronic disease self-management. Qualitative data were submitted to thematic content analysis. Results: $93.7 \%$ of participants present comorbidities, mainly cardiovascular and endocrine diseases. Three themes emerged from the interviews: Mastery in the management of medications; Difficulty in adopting a stable, healthy behaviour; Importance of a close and trusting relationship with health professionals. Conclusion: The self-management needs of older people that can be diminished through nursing interventions were highlighted, emphasising areas to be intervened and strategies to be used in programmes aimed at promoting the health literacy of this population.
\end{abstract}

DESCRIPTORS: Elderly; Self-management; Chronic Disease; Ageing; Nursing.

COMO REFERENCIAR ESTE ARTIGO:

Bastos C, Santos C, Martins MM, Fernandes C, Lima L. Necessidades dos idosos na autogestão da doença crônica: perspectivando um programa de intervenção de enfermagem. Cogit. Enferm. [Internet]. 2021 [acesso em "colocar data de acesso, dia, mês abreviado e ano"]; 26. Disponível em: http://dx.doi.org/10.5380/ce.v26i0.83048. 
O envelhecimento da população mundial tem sido um fenômeno crescente nos últimos anos ${ }^{(1-2)}$. Em Portugal, o grupo de pessoas com 65 ou mais anos representa $22 \%$ da população atual, e o índice de envelhecimento (número de idosos por 100 jovens) é de $161^{(3)}$. Apesar de as pessoas viverem mais anos, nem sempre esses anos são vividos com saúde. O fenômeno do envelhecimento é acompanhado pelo aumento das doenças crônicas ${ }^{(4)}$, que têm o potencial de limitar o estado funcional, a produtividade e a qualidade de vida das pessoas que com elas vivem ${ }^{(5)}$.

O envelhecimento implica um aumento do risco de desenvolvimento de vulnerabilidades, as quais envolvem um conjunto de aspectos individuais e coletivos que exercem influência nas condições de vida e de saúde ${ }^{(6)}$, e tornam os idosos um grupo particular com necessidade de apoio para viver com mais saúde e reduzir o impacto das doenças crônicas. Devem assim ser planejados, desenvolvidos e implementados programas e intervenções comunitárias para pessoas com doenças crônicas, conciliando esforços do sistema de cuidados de saúde, das organizações sociais e da comunidade acadêmica ${ }^{(5)}$.

No âmbito de um projeto de investigação mais amplo denominado "PT4Ageing: Personal Trainer para a gestão da saúde de pessoas com mais idade", será desenvolvido um programa de intervenção centrado na autogestão da doença crônica. Uma vez que em Portugal é escassa a evidência neste domínio, foi decidido desenvolver um primeiro estudo de caracterização da população alvo.

O fenômeno emergente do envelhecimento populacional desafia a implementação de políticas sustentáveis na sociedade atual e vindoura ${ }^{(7)}$, promotoras do envelhecimento saudável|(1-2). O envelhecimento saudável é um processo ao longo da vida e é mais do que a ausência de doença $a^{(2)}$. No entanto, a par do envelhecimento e das mudanças dos estilos de vida, há um crescimento das doenças crônicas $^{(4,8)}$, as quais se associam ao aumento da morbidade e da mortalidade, particularmente visível nas pessoas mais idosas ${ }^{(9)}$.

De acordo com o "Retrato da Saúde 2018"(10), atualmente em Portugal vive-se mais anos, mas com um menor número de anos de vida saudável depois dos 65 anos de idade, quando comparado com os restantes países da Organização para a Cooperação e Desenvolvimento (OCDE). Os idosos em Portugal apresentam mais comorbidades, destacando-se a diabetes, doenças cardiovasculares, obesidade e doenças oncológicas ${ }^{(10)}$.

Os idosos com problemas de saúde necessitam de receber mais cuidados de saúde e suporte social(7). Em Portugal, a população idosa possui também características sociodemográficas que a tornam particularmente vulnerável, destacando-se a baixa literacia, o baixo poder econômico e a falta de acesso a estruturas sociais, que substituam o apoio às necessidades físicas e psicossociais, numa etapa da vida em que naturalmente as fontes de suporte informal próximas vão desaparecendo ${ }^{(3,10)}$. Face à existência de comorbidades com necessidade de polimedicação, a gestão da doença nesta faixa etária torna-se ainda mais complexa ${ }^{(10)}$. O papel dos profissionais de saúde neste domínio é crucial, destacandose, entre estes, a intervenção dos enfermeiros.

A Organização Mundial de Saúde identifica a necessidade de novos modelos de promoção da saúde e prevenção da doença para os idosos (2). Em um estudo de revisão, são identificados programas de autogestão eficazes, produzindo resultados modestos, mas clinicamente significativos, a nível de indicadores como comportamentos de saúde, estatuto de saúde, autoeficácia e qualidade de vida, e ainda a diminuição do recurso a cuidados de saúde ${ }^{(11)}$. Evidentemente que a eficácia destes programas depende da identificação prévia das necessidades específicas das populações a que se destinam. Num estudo qualitativo desenvolvido nos Estados Unidos da América sobre a vivência de idosos com múltiplas doenças crônicas, foram identificadas várias necessidades, tais como dificuldades emocionais associadas aos sintomas (por exemplo, ansiedade e tristeza), 
implicações funcionais das doenças que ameaçavam o bem-estar e fatores relacionados com os cuidados de saúde, nomeadamente dificuldades na obtenção de informação ou dificuldades na comunicação com os profissionais de saúde ${ }^{(12)}$.

Para a caracterização da população idosa em relação à gestão da doença crônica, para além de indicadores clínicos, tais como a presença de patologias e sintomas, é importante a avaliação da sua percepção acerca da sua saúde e do processo de autogestão da doença. Impõe-se a recolha de dados que reflitam as experiências dos idosos nestes domínios e que permitam identificar as necessidades para as quais os enfermeiros podem constituir um recurso. As autopercepções dos idosos são multifacetadas e complexas, podendo influenciar o bem-estar e a qualidade de vida ${ }^{(13)}$.

Segundo um estudo de revisão, os programas de apoio aos idosos nem sempre usam estratégias para lidar com o impacto das doenças na sua vida diária, pelo que os autores salientam que os programas necessitam se adaptar às necessidades dos idosos com múltiplas doenças e avaliar se os participantes estão a utilizar as estratégias propostas ${ }^{(14)}$.

Em Portugal, existe escassa produção científica nesta área e, previamente ao desenvolvimento do programa, considerou-se oportuno desenvolver um estudo de caracterização da população idosa com doença crônica. Assim, os objetivos deste estudo são caracterizar o perfil de saúde de idosos da comunidade e identificar os domínios do processo de autogestão da doença crônica para intervenções de enfermagem.

\section{MÉTODO}

Trata-se de um estudo misto, entre os meses de setembro de 2018 e julho de 2019, em uma unidade de saúde da região urbana do norte de Portugal. Foi constituída uma amostra não probabilística de conveniência, recrutada em instituições de saúde da cidade do Porto, composta por 48 idosos da comunidade.

Os critérios de inclusão foram: 65 ou mais anos de idade; residentes na comunidade; portadores de, pelo menos, uma doença crônica; autônomos na deslocação à unidade de saúde; sem déficit cognitivo. Todos os idosos responderam a um formulário, mas apenas quatro deles participaram da entrevista semiestruturada. As entrevistas e a aplicação do formulário realizaram-se nas unidades de saúde, em ambiente privado, por colaboradores que receberam treino previamente à recolha dos dados.

Para a recolha dos dados, foram congregados diversos instrumentos, designadamente de caracterização sociodemográfica e clínica, autopercepção de saúde, perfil do estilo de vida individual, escala de adesão aos tratamentos, inventário de avaliação da qualidade de vida e guião da entrevista.

O instrumento de caracterização sociodemográfica e clínica, construído especificamente para o presente estudo, avalia variáveis como sexo, estado civil, escolaridade e presença de patologias e sintomas. A autopercepção de saúde é avaliada por uma questão: "Em geral, diria que a sua saúde é?", pontuada numa escala de resposta de tipo Likert com cinco pontos: "um=Má"; "dois=Aceitável"; "três=Boa"; "quatro=Muito Boa"; "cinco=Excelente".

O perfil do estilo de vida individual, adaptado para o presente estudo, é composto pelas subescalas "Alimentação"; "Atividade física"; "Comportamento preventivo"; "Relacionamento social"; e "Controle do stress". A consistência interna da escala global é de 0,91 . Possui uma escala de respostas de tipo Likert com quatro pontos; quanto maior o valor final, maior é a adesão ao estilo de vida específico.

A Medida de Adesão aos Tratamentos (MAT) é uma escala desenvolvida por Morisky, 
Green e Levine em 1986, que foi traduzida e adaptada para o idioma português ${ }^{(15)}$. Esta escala é composta por sete itens, em que o respondente é questionado relativamente às vezes que tem determinado comportamento relativamente aos medicamentos que lhe foram prescritos. As respostas são apresentadas numa escala de tipo Likert de seis pontos, que se estendem entre o "um=Sempre"; a "seis= Nunca". Na leitura dos resultados, a um maior valor corresponde uma melhor adesão à medicação prescrita. A fiabilidade da Escala MAT no presente estudo foi de 0,682.

O Inventário de Avaliação da Qualidade de Vida em Adultos Idosos da Organização Mundial de Saúde (WHOQ̧OL-OLD), na sua versão portuguesa(16), é composto por 28 questões, agrupadas em sete subescalas/domínios: "Funcionamento sensorial"; "Autonomia"; "Atividades passadas, presentes e futuras"; "Participação Social"; "Morte e morrer"; "Intimidade"; "Família/Vida familiar". A Escala Global (28 itens) apresentou, no presente estudo, uma consistência interna de 0,904. As respostas são dadas em escalas de concordância de tipo Likert e, quanto maior o valor obtido, melhor é a percepção de qualidade de vida.

A entrevista semiestruturada, seguiu um guia que incluiu várias questões abertas, centradas na descrição do estado de saúde, processo de gestão da doença, estilos de vida e necessidades sentidas no âmbito dos cuidados de enfermagem centrados na autogestão da doença crônica.

Os dados quantitativos foram analisados de forma descritiva com recurso ao software SPSS Statistics, versão 25.0. Os resultados são apresentados através de frequência absoluta e relativa, média e desvio padrão. As entrevistas, depois de transcritas, foram submetidas a uma análise temática ${ }^{(17)}$.

O presente estudo foi aprovado pela Comissão de Ética da Administração Regional de Saúde da região Norte de Portugal em janeiro de 2018 (Parecer n⿳1/2018). Os participantes foram esclarecidos sobre os objetivos e finalidade do estudo e foi-lhes pedido o preenchimento e assinatura do consentimento informado.

\section{RESULTADOS}

Relativamente aos participantes, a amostra é composta majoritariamente por pessoas do sexo feminino $(n=32 ; 67 \%)$, casadas ou em união estável $(n=27 ; 56 \%)$, e com idade entre os 65 e os 90 anos, com uma média de 74,9 anos (DP=6,80).

A escolaridade média da amostra é baixa $(M=3,65$ anos; $D P=3,82)$, com um mínimo de zero e um máximo de 18 anos. Os participantes estão, na sua grande maioria, reformados $(n=42 ; 88 \%)$, tendo exercido diferentes tipologias de atividade profissional, de acordo com a Classificação Nacional das Profissões de 2010. Dois idosos, no entanto, referiram ainda se manter na situação de trabalho $(4,2 \%)$. O agregado familiar dos idosos teve uma amplitude de possuir entre um a cinco elementos e era, em média, composto por 1,98 (DP=1,06), e apenas um $(2,1 \%)$ participante vivia sozinho.

Dando resposta ao objetivo de caracterizar o estado de saúde de idosos residentes na comunidade, a amostra apresenta um conjunto de doenças crônicas, a grande maioria com comorbidades $(n=45 ; 94 \%)$. De notar que 24 participantes, correspondendo a $50 \%$ da amostra, têm duas doenças associadas. As doenças crônicas mais comuns são as cardiovasculares e as endócrinas. Como esperado, a amostra relatou um conjunto de sintomas, sendo mais comuns as dores musculoesqueléticas e osteoarticulares, seguidos da diminuição da visão.

Dos 16 respondentes (33,3\%) que indicaram ter dor/moedeira ou desconforto, no momento da recolha de dados, esta situava-se em uma ou várias partes do corpo 
e com uma intensidade média de 4,68 (DP=3,19), avaliada através da Escala de Faces, que corresponde a uma "dor moderada". Nota-se, no entanto, que sete doentes $(14,7 \%)$ avaliaram a sua dor como intensa (valores de sete, oito e nove na escala). A medicação é a inerente às suas doenças crônicas, a maioria tomando entre dois e três fármacos em associação, resultando em polimedicação, observável na Tabela 1.

Tabela 1 - Caracterização sociodemográfica e clínica dos participantes (n=48). Porto, Portugal, 2019 (continua)

\section{Variáveis}

n $\%$

Sexo

\begin{tabular}{|c|c|c|}
\hline Masculino & 16 & 33,3 \\
\hline Feminino & 32 & 66,7 \\
\hline \multicolumn{3}{|l|}{ Idade } \\
\hline $65-74$ anos & 29 & 60,4 \\
\hline $75-84$ anos & 15 & 31,3 \\
\hline$>85$ anos & 4 & 8,3 \\
\hline \multicolumn{3}{|l|}{ Estado civil } \\
\hline Casado(a)/união de facto & 27 & 56,3 \\
\hline Viúvo(a) & 15 & 31,3 \\
\hline Separado/Divorciado & 4 & 8,3 \\
\hline Solteiro & 2 & 4,2 \\
\hline \multicolumn{3}{|l|}{ Escolaridade } \\
\hline Sem escolaridade ( 0 anos) & 15 & 31,3 \\
\hline $1^{\circ}$ Ciclo ( $1^{a}$ à $4^{a}$ classe) & 25 & 52,1 \\
\hline $2^{\circ}$ Ciclo $\left(5^{\circ}\right.$ ao $6^{\circ}$ ano $)$ & 3 & 6,3 \\
\hline $3^{\circ}$ Ciclo $\left(7^{\circ}\right.$ ao $9^{\circ}$ ano $)$ & 2 & 4,2 \\
\hline Ensino Secundário $\left(10^{\circ}\right.$ ao $12^{\circ}$ ano $)$ & 1 & 2,1 \\
\hline Ensino Superior (>12 anos) & 2 & 4,2 \\
\hline \multicolumn{3}{|l|}{ Número de elementos agregado familiar } \\
\hline 0 & 1 & 2,1 \\
\hline 1 & 17 & 35,4 \\
\hline 2 & 18 & 37,5 \\
\hline$>3$ & 12 & 25 \\
\hline \multicolumn{3}{|l|}{ Doença associadas } \\
\hline Doença cardiovascular (enfarte, angina de peito, HTA) & 36 & $75 \%$ \\
\hline Doença endócrina (diabetes mellitus) & 25 & 52,1 \\
\hline Doença musculoesquelética e osteoarticular (artrite/artrose) & 21 & 43,8 \\
\hline Doença psiquiátrica (depressão) & 7 & 14,6 \\
\hline Doença respiratória (DPOC, asma, bronquite) & 6 & 12,5 \\
\hline
\end{tabular}




\begin{tabular}{|c|c|c|}
\hline Doença cerebrovascular (AVC) & 4 & 8,3 \\
\hline Doença oncológica & 1 & 2,1 \\
\hline $\begin{array}{l}\text { Outras doenças (oftálmicas, gastrointestinais, metabólicas - tiroide; } \\
\text { neurológicas - epilepsia) }\end{array}$ & 8 & 16,7 \\
\hline \multicolumn{3}{|l|}{ Doenças em associação (comorbilidades) } \\
\hline 0 & 3 & 6,3 \\
\hline 1 & 6 & 12,5 \\
\hline 2 & 24 & 50 \\
\hline$>3$ & 15 & 31,3 \\
\hline \multicolumn{3}{|l|}{ Sintomas } \\
\hline Dor musculoesquelética e osteoarticular (artrite/artrose) & 26 & 54,2 \\
\hline Diminuição da visão & 25 & 52,1 \\
\hline Dor/moedeira ou desconforto & 16 & 33,3 \\
\hline Dificuldade em andar & 15 & 31,3 \\
\hline Sentimentos de tristeza persistente & 10 & 20,8 \\
\hline Desequilíbrios constantes & 10 & 20,8 \\
\hline Perdas de urina/incontinência urinária & 8 & 16,7 \\
\hline Alterações de memória que interferem na vida do dia a dia & 6 & 12,5 \\
\hline Sem sintomas & 10 & 20,8 \\
\hline \multicolumn{3}{|l|}{ Medicação } \\
\hline Medicamentos para o sistema cardiovascular & 45 & 93,8 \\
\hline Antidiabéticos orais & 24 & 50 \\
\hline Analgésicos & 10 & 20,8 \\
\hline Anti-inflamatórios & 9 & 18,8 \\
\hline Antidepressivos & 9 & 18,8 \\
\hline Insulina & 6 & 12,5 \\
\hline Sedativos & 5 & 10,4 \\
\hline Medicamentos para o sistema respiratório & 4 & 8,3 \\
\hline \multicolumn{3}{|l|}{ Total de medicamentos (em associação) } \\
\hline 1 & 10 & 20,8 \\
\hline 2 & 19 & 39,6 \\
\hline 3 & 12 & 25 \\
\hline$>3$ & 6 & 12,5 \\
\hline
\end{tabular}

Fonte: Autores (2019).

Tendo por base os objetivos do estudo, considerou-se adequado calcular as medidas descritivas de algumas variáveis, designadamente autopercepção de saúde, adesão aos tratamentos (MAT), estilos de vida e qualidade de vida (WHOQOL-OLD), apresentados na Tabela 2. 
Tabela 2 - Variáveis em análise. Porto, Portugal, 2019

Variáveis n M DP Med. Moda Min. Max.

\begin{tabular}{|c|c|c|c|c|c|c|c|c|}
\hline \multicolumn{2}{|c|}{ Autopercepção de Saúde } & 48 & 2,54 & 0,82 & 2 & 2 & 1 & 5 \\
\hline \multicolumn{2}{|c|}{ Adesão aos tratamentos (MAT) } & 48 & 5,69 & 0,4 & 5,86 & 6 & 4 & 6 \\
\hline \multirow{5}{*}{$\begin{array}{l}\text { Estilos de } \\
\text { vida }\end{array}$} & Alimentação & 48 & 1,9 & 0,73 & 2 & 2 & 0 & 3 \\
\hline & Atividade física & 48 & 0,82 & 0,92 & 0,5 & 0 & 0 & 3 \\
\hline & Comportamento preventivo & 45 & 2,16 & 0,69 & 2,2 & 2 & 0 & 3 \\
\hline & Relacionamento social & 48 & 1,72 & 0,79 & 1,67 & 1 & 0 & 3 \\
\hline & Controle do Stress & 48 & 1,82 & 0,68 & 2 & 0 & 1 & 3 \\
\hline \multirow{8}{*}{$\begin{array}{l}\text { Qualidade } \\
\text { de Vida } \\
\text { (WHOQOL- } \\
\text { OLD) }\end{array}$} & Funcionamento Sensorial & 47 & 16,26 & 3,14 & 17 & 19 & 9 & 20 \\
\hline & Autonomia & 47 & 17,81 & 2,11 & 18 & 20 & 12 & 20 \\
\hline & $\begin{array}{l}\text { Atividades Passadas } \\
\text { Presentes e futuras }\end{array}$ & 46 & 15,46 & 2,86 & 15 & 15 & 8 & 20 \\
\hline & Participação social & 48 & 15,48 & 3,2 & 15,5 & 16 & 8 & 20 \\
\hline & Morte e morrer & 47 & 14,34 & 4,03 & 15 & 16 & 4 & 20 \\
\hline & Intimidade & 48 & 15,58 & 4,56 & 16 & 20 & 4 & 20 \\
\hline & Família/Vida Familiar & 48 & 16,58 & 3,4 & 17 & 20 & 8 & 20 \\
\hline & Global & 44 & 111,34 & 14,93 & 110,5 & 109 & 75 & 137 \\
\hline
\end{tabular}

Fonte: Autores (2019).

No que se refere à autopercepção de saúde, os resultados indicam que a amostra tinha uma percepção situada entre "Aceitável" e "Boa". De notar, porém, que 50\% da amostra a classificou como "Aceitável" e 4,2\% como "Má". Relativamente à adesão à medicação, foi encontrada uma média de 5,69 (DP=0,40). Considerando o valor da mediana $(5,86)$, a amostra, na sua globalidade, revela uma adesão elevada à medicação prescrita.

Os participantes demonstraram ainda adotar um estilo de vida saudável, essencialmente, para o comportamento do tipo preventivo, seguido da alimentação saudável. Em contrapartida, parecem aderir pouco à atividade física. Globalmente, os idosos que integraram a amostra percepcionam a sua qualidade de vida como razoável/ moderada. No que se refere às subescalas/domínios, os resultados mostram que a amostra percepciona uma boa "Autonomia" e ainda um bom "Funcionamento Sensorial", assim como uma boa qualidade de vida na "Família/Vida familiar". Em contrapartida, manifesta uma baixa percepção de qualidade de vida no que se refere à "Morte e Morrer".

De modo a explorar os domínios do processo de autogestão da doença crônica que requerem intervenção de enfermagem, e com recurso a entrevistas realizadas com quatro participantes, destacaram-se os seguintes temas: "Domínio na gestão da medicação", "Dificuldade em se comportar sempre de forma saudável", "Importância de uma relação próxima e de confiança com os profissionais de saúde".

Relativamente ao primeiro tema "Domínio na gestão da medicação", todos os participantes partilharam esse sentimento, afirmando conhecer toda a medicação e revelando facilidade em cumpri-la, nomeadamente em relação aos horários e formas de administração. Foi ainda partilhada a necessidade de fazer ajustes ao regime medicamentoso, que embora fossem de iniciativa dos participantes, eram posteriormente validados pelo médico. Os depoimentos descrevem essas afirmações: 
Eu não estou com esses medicamentos para o colesterol... aconselharam-me as pastilhas de arroz vermelho... como não é uma coisa química... Mas eu já disse à senhora doutora e ela sabe disso perfeitamente. (P1)

O médico mandou-me fazer uma de manhã em jejum e outra à noite. Mas como eu metia muita insulina, se eu me esquecesse de comer alguma coisa de manhã eu quase que caía. Então eu por minha iniciativa comecei a dividir a insulina... e dividi por três vezes. Faço isto em jejum, antes do almoço, e antes do jantar. E nunca mais tive problemas ...e eu disse ao médico ...eu experimentei, assim assim, e ele disse: "o senhor dá-se bem? Então continue". Até hoje. (P3)

O segundo tema "Dificuldade em se comportar sempre de forma saudável" esteve muito presente nos relatos dos quatro entrevistados e descreve a exigência do cumprimento de comportamentos de saúde normalmente presentes na componente não farmacológica do regime terapêutico, principalmente no que diz respeito à alimentação, ao exercício físico e à gestão emocional. É de notar a referência às alterações decorrentes do envelhecimento, como fatores mencionados como dificultando o cumprimento de algumas orientações de saúde.

...chegamos assim a esta idade, tudo nos sabe bem. $E$ às vezes exageramos um bocado e não devíamos. Eu tenho alturas que tenho a certeza que faço asneiras. Eu sei que devia comer menos até do que aquilo que como porque eu engordei seis quilos. Não devia. Mas prontos... (P3)

...às vezes eu abuso que, agora nesta idade, a gente torna-se mais lambareira. (P4)

Quanto ao terceiro tema "Importância de uma relação próxima e de confiança com os profissionais de saúde", que foi o mais desenvolvido pelos participantes, é de referir a menção à importância da confiança depositada na competência técnica dos profissionais de saúde no que diz respeito ao apoio nas decisões terapêuticas ao longo do processo de gestão da doença.

É o dar atenção, explicar, dar atenção... Se a pessoa chega aqui... ter um bocado de atenção com ela. (P2)

Eu acho que o mais importante de uma enfermeira é elucidar os doentes.... Dizer ao doente o que tem, o que não tem, o que deve tomar, educar o doente. Para mim é o mais importante. Essas pessoas precisam de ajuda de informação. De explicar... olhar bem para elas é muito importante. (P3)

Não menos importante, a referência aos aspectos interpessoais e à atenção individualizada que os participantes mencionaram como promotora do seu sentimento de segurança e de bem-estar, principalmente numa etapa da vida que estes encaram como mais suscetível ao isolamento e à solidão.

Se houvesse uma visita, ou coisa do gênero... Agora o que eu acho é que a vida é cada vez mais isolada... eu acho que isso é mais fundamental do que até a medicação. (P2)

Oaumento do número de pessoas idosas, que vivem com doenças crônicas, representa um problema de saúde pública de crescente importância. A autogestão é uma estratégia promissora, enfatizando a responsabilidade do doente no processo de cuidados. Vai além da educação do doente, ensinando a identificar ativamente os problemas associados à doença e a resolvê-los, ou procurar ajuda. Inclui lidar com sintomas e incapacidade; monitorização de indicadores; gestão complexa de medicação; manutenção de níveis 
adequados de nutrição, dieta e exercício; ajuste às exigências psicológicas e sociais, incluindo ajustes de estilo de vida; e participação eficaz nas interações com os cuidados de saúde ${ }^{(18)}$.

Apesar de todos os idosos que participaram deste estudo serem portadores de uma ou mais doenças crônicas e de sintomatologia que afetam áreas muito importantes da funcionalidade, a saúde percebida descrita foi satisfatória, assim como a sua percepção de qualidade de vida. Os resultados de todas as subescalas e escala total de qualidade de vida enquadram-se dentro de parâmetros satisfatórios ou bons, tal como verificado num outro estudo realizado com idosos ${ }^{(19)}$. Verificou-se que os valores são também superiores aos obtidos no estudo de validação do instrumento com uma amostra de idosos da comunidade ${ }^{(16)}$. De todos os domínios, aquele em que os valores médios foram mais elevados foi o relativo à autonomia, valor este que é também o que mais se destaca, pela positiva, nos valores encontrados em outro estudo(16). A forma como os idosos descreviam a sua experiência no domínio da gestão da doença era também reveladora de que estes continuavam a sentirem-se independentes e a funcionar globalmente de forma autossuficiente.

Entre possíveis explicações, pode-se apontar o fato de que algumas das patologias mais frequentes, como as doenças osteoarticulares, serem consideradas associadas ao processo de envelhecimento, e talvez por esta razão, os idosos se vão adaptando mais facilmente às suas limitações, desenvolvendo estratégias compensatórias e aferindo as suas expectativas em relação à funcionalidade.

Quanto à gestão do regime terapêutico, os resultados obtidos através da escala de adesão ao tratamento foram elevados, reveladores de uma boa adesão ao tratamento medicamentoso. No mesmo sentido, e nas entrevistas, os idosos relataram um sentido de elevada competência na gestão da medicação, tendo, no entanto, também descrito situações em que contrariamente ao que os resultados quantitativos fazem supor, fizeram alterações na administração e doses da medicação, ou interrompido um tratamento prescrito pelo médico, substituindo-o por uma alternativa que consideraram mais natural.

Apesar de o instrumento MAT ser muito utilizado na investigação, quer a nível nacional quer internacional, o fato de este produzir quase sempre resultados bastante elevados, mesmo junto de populações idosas, leva à hipótese de um possível efeito de teto. Neste sentido, os resultados apontam para a necessidade de as intervenções no âmbito da autogestão contemplarem esta área, nomeadamente no conhecimento e competências necessárias às tomadas de decisão relativas à medicação.

Em relação à componente não farmacológica do regime terapêutico, avaliada neste estudo através da escala de estilos de vida e explorada nas entrevistas, mais uma vez temos resultados que não são totalmente coincidentes. O exercício físico foi o domínio em que os valores médios na escala foram mais baixos, resultado que vai ao encontro de outros estudos ${ }^{(12)}$. Apesar de, na resposta à escala de estilos de vida, os entrevistados terem relatado comportamentos alimentares saudáveis, nas entrevistas foram partilhadas dificuldades na manutenção deste tipo de comportamento.

Os dados revelam diferenças entre os dados quantitativos e qualitativos, alguns deles contraditórios. Conforme referem estes autores, notavelmente, a autoeficácia é um resultado intermediário importante, que mede a crença de um indivíduo na sua capacidade de realizar certas tarefas; mas não avalia o uso de estratégias ou se as tarefas de autogestão são concluídas ${ }^{(14)}$. Impõe-se, assim, a necessidade de explorar e construir novos programas, que ajudem a sustentar mudanças de comportamento para melhorar os resultados de saúde e reduzir o uso de serviços de saúde pelas pessoas com condições crônicas $^{(11)}$. Devem ser consideradas estratégias úteis e integrá-las nas rotinas de vida diária dos participantes, ou será improvável uma mudança de comportamento positiva e sustentáve|(14).

Neste sentido, o desenho da intervenção a desenvolver, impõe que sejam trabalhadas 
áreas que são domínios normalmente presentes nos programas de autogestão e que, neste estudo, emergiram também como relevantes para a população alvo: viver com uma condição crônica (controle da doença e tomada de decisão); adesão à medicação; alimentação saudável e exercício físico; gestão de stress/ansiedade e relaxamento; relações interpessoais; e comunicação com os profissionais de saúde.

No âmbito deste estudo, principalmente na sua componente qualitativa, foi reforçada a crença de que a metodologia a adotar na intervenção será a de trabalho em pequenos grupos, de oito a 12 idosos, com o recurso a atividades que, além de fomentar o desenvolvimento das capacidades e da autoeficácia dos participantes na gestão das suas condições crônicas, será um espaço para a promoção do suporte mútuo. A facilitação das sessões por profissionais da área da saúde (enfermeiros e psicólogo) constituirá também uma oportunidade para experimentar e ensaiar a comunicação com os profissionais e, mais importante, estabelecer relações em que, como sugere um dos participantes, a atenção é individualizada e responde às necessidades de cada um: "Para mim é o mais importante. Essas pessoas precisam de ajuda de informação. De explicar... olhar bem para elas é muito importante."

Pretende-se, com a aplicação do programa de autogestão das doenças crônicas, promover o desenvolvimento da autoeficácia e sentido de coerência entre os idosos, podendo este ser usado na sua capacitação para alcançar a compreensibilidade, a melhor capacidade de gestão do seu regime terapêutico e um melhor significado para as suas vidas $^{(20)}$.

Como limitações do estudo, aponta-se a escassez de estudos nacionais nesta área e o tamanho reduzido da amostra, o que não permite a comparação e a generalização dos resultados apresentados.

\section{CONCLUSÃO}

Este estudo permitiu identificar o perfil de saúde e as necessidades dos idosos no que diz respeito à autogestão da doença crônica, constituindo um contributo importante para o desenvolvimento de um programa de autogestão da doença crônica, no âmbito do projeto "PT4Ageing: Personal Trainer para a gestão da saúde de pessoas com mais idade".

Apesar de os participantes se perceberem com boa saúde e qualidade de vida, os resultados evidenciam algumas lacunas na gestão das suas doenças crônicas, que os enfermeiros e demais profissionais de saúde podem ajudar a desenvolver e aperfeiçoar, promovendo assim uma melhor literacia em saúde. Emergiram ainda deste estudo as áreas mais relevantes a serem trabalhadas no programa de intervenção a dirigir a esta população, nomeadamente: viver com uma condição crônica (controle da doença e tomada de decisão); adesão à medicação; alimentação saudável e exercício físico; gestão de stress/ansiedade e relaxamento; relações interpessoais e comunicação com os profissionais de saúde.

\section{AGRADECIMENTOS}

Este artigo foi apoiado pelo FCT - Fundação para a Ciência e a Tecnologia, I.P., CINTESIS, R\&D Unit (referência UIDB/4255/2020). 
1. World Health Organization (WHO). World report on ageing and health. [Internet]. Geneva: WHO; 2015 [acesso em 05 maio 2020]. Disponível em: https://apps.who.int/iris/handle/10665/186463.

2. World Health Organization (WHO). Global strategy and action plan on ageing and health. [Internet]. Geneva: WHO; 2017 [acesso em 07 maio 2020]. Disponível em: https://www.who.int/ageing/WHOGSAP-2017.pdf.

3. PORDATA. Fundação Francisco Manuel dos Santos. Retrato de Portugal - Edição 2020. [Internet]. PORDATA; 2020. [acesso em 07 maio 2020]. Disponível em: https://www.pordata.pt/ebooks/ PT2020v20200710/mobile/index.html.

4. Suzman R, Beard JR, Boerma T, Chatterji S. Health in an ageing world-what do we know? Lancet. [Internet]. 2015 [acesso em 11 maio 2020]; 385(9967). Disponível em: https://doi.org/10.1016/S01406736(14)61597-X.

5. Institute of Medicine. Living well with chronic illness: a call for public health action. Military Medicine. [Internet]. 2015 [acesso em 05 maio 2020]; 180(5). Disponível em: https://doi.org/10.7205/ MILMED-D-15-00034.

6. Barbosa KTF, Oliveira FMRL de, Fernandes M das GM. Vulnerability of the elderly: a conceptual analysis. Rev. Bras. Enferm. [Internet]. 2019 [acesso em 05 maio 2020]; 72(suppl 2). Disponível em: https:// doi.org/10.1590/0034-7167-2018-0728.

7. Bloom DE, Chatterji S, Kowal P, Lloyd-Sherlock P, McKee M, Rechel B, et al. Macroeconomic implications of population ageing and selected policy responses. Lancet. [Internet]. 2015 [acesso em 05 maio 2020]; 385(9968). Disponível em: https://doi.org/10.1016/S0140-6736(14)61464-1.

8. Åberg C, Gillsjö C, Hallgren J, Berglund M. "It is like living in a diminishing world": older persons' experiences of living with long-term health problems - prior to the STRENGTH intervention. Int J Qual Stud Health Well-being. [Internet]. 2020 [acesso em 05 maio 2020]; 15(1). Disponível em: https://doi.org/1 $\underline{0.1080 / 17482631.2020 .1747251 .}$.

9. Prince MJ, Wu F, Guo Y, Robledo LMG, O'Donnell M, Sullivan R, et al. The burden of disease in older people and implications for health policy and practice. Lancet. [Internet]. 2015 [acesso em 05 maio 2020]; 385(9967). Disponível em: https://doi.org/10.1016/S0140-6736(14)61347-7.

10. República Portuguesa. Serviço Nacional de Saúde. Retrato da Saúde 2018. [Internet]. SNS: Portugal; 2018. [acesso em 05 maio 2020]. Disponível em: https://www.sns.gov.pt/wp-content/uploads/2018/04/ RETRATO-DA-SAUDE 2018 compressed.pdf.

11. Allegrante JP, Wells MT, Peterson JC. Interventions to Support Behavioral Self-Management of Chronic Diseases. Annu Rev Public Health. [Internet]. 2019 [acesso em 05 maio 2020]; 40. Disponível em: https://doi.org/10.1146/annurev-publhealth-040218-044008.

12. Thomas-Purcell KB, Jacobs RJ, Seidman TL, Acevedo A, Waldrop-Valverde D, Ownby RL. A mixed analysis approach to elucidate the multiple chronic condition experience of English- and Spanish-speaking older adults. Clin Interv Aging. [Internet]. 2019 [acesso em 15 abril 2020]; 14. Disponível em: https://doi. org/10.2147/CIA.S193215.

13. Hausknecht S, Low LF, O'Loughlin K, McNab J, Clemson L. Older Adults' Self-Perceptions of Aging and Being Older: a Scoping Review. Gerontologist. [Internet]. 2020 [acesso em 15 abril 2020]; 60(7). Disponível em: https://doi.org/10.1093/geront/gnz153.

14. Warner G, Packer TL, Kervin E, Sibbald K, Audulv Å. A systematic review examining whether community-based self-management programs for older adults with chronic conditions actively engage participants and teach them patient-oriented self-management strategies. Patient Educ Couns. [Internet]. 2019 [acesso em 15 abril 2020]; 102(12). Disponível em: https://doi.org/10.1016/j.pec.2019.07.002.

15. Delgado $A B$, Lima ML. Contributo para a validação concorrente de uma medida de adesão aos tratamentos. Psicologia, Saúde \& Doenças. [Internet]. 2001 [acesso em 18 abril 2020]; 2(2). Disponível em: https://repositorio.ispa.pt/bitstream/10400.12/1114/1/PSD\%202001\%20\%282\%292\%2081-100.pdf. 
16. Vilar M, Sousa LB, Simões MR. The European Portuguese WHOQOL-OLD module and the new facet Family/Family life: reliability and validity studies. Qual Life Res. [Internet]. 2016 [acesso em 15 abril 2020]; 25. Disponível em: https://doi.org/10.1007/s11136-016-1275-9.

17. Braun V, Clarke V. Using thematic analysis in psychology. Qualitative Research in Psychology. [Internet]. 2006 [acesso em 15 abril 2020]; 3(2). Disponível em: https://doi.org/10.1191/1478088706qp063oa.

18. Grady PA, Gough LL. Self-Management: a comprehensive approach to management of chronic conditions. Am J Public Health. [Internet]. 2014 [acesso em 15 abril 2020]; 108. Disponível em: https://doi. org/10.2105/AJPH.2014.302041.

19. Lima LMM, Santos CSV de B, Almeida MCBM de, Martins MMFP da S. Psychosocial adjustment and health in older people: cluster analysis. Referência. [Internet]. 2018 [acesso em 15 abril 2020]; 4(16). Disponível em: https://doi.org/10.12707/RIV17070.

20. Hourzad A, Pouladi S, Ostovar A, Ravanipour M. The effects of an empowering self-management model on self-efficacy and sense of coherence among retired elderly with chronic diseases: a randomized controlled trial. Clin Interv Aging. [Internet]. 2018 [acesso em 15 abril 2020]; 2018. Disponível em: https:// doi.org/10.2147/CIA.S183276. 


\section{NECESSIDADES DOS IDOSOS NA AUTOGESTÃO DA DOENÇA CRÔNICA: PERSPECTIVANDO UM PROGRAMA DE INTERVENÇÃO DE ESNERMAGEM}

\section{RESUMO:}

Objetivo: caracterizar o perfil de saúde de idosos da comunidade e identificar os domínios do processo de autogestão da doença para intervenções de enfermagem. Método: estudo misto, integrando 48 participantes de Porto, Portugal entre setembro/2018 e julho/2019. Foi utilizado um formulário para avaliação de doenças e variáveis relacionadas com a saúde, e uma entrevista semiestruturada centrada na autogestão da doença crônica. Os dados qualitativos foram submetidos à análise de conteúdo temática. Resultados: 93,7\% dos participantes apresentam comorbidades, destacando-se as doenças cardiovasculares e endócrinas. Das entrevistas, emergiram três temas: Domínio na gestão de medicamentos; Dificuldade de se comportar sempre de forma saudável; Importância de uma relação estreita e de confiança com os profissionais de saúde. Conclusão: evidenciaram-se as necessidades de autogestão das pessoas idosas que podem ser minimizadas com intervenções dos enfermeiros, destacando as áreas a intervir e estratégias a utilizar nos programas dirigidos à promoção da saúde.

DESCRITORES: Idoso; Autogestão; Doença crônica; Envelhecimento; Enfermagem.

\section{NECESIDADES DE LOS ANCIANOS EN EL AUTOMANEJO DE LA ENFERMEDAD CRÓNICA: DISEÑO DE UN PROGRAMA DE INTERVENCIÓN DE ENFERMERÍA}

\section{RESUMEN:}

Objetivo: caracterizar el perfil de salud de las personas mayores en la comunidad e identificar los dominios del proceso de automanejo de la enfermedad para las intervenciones de enfermería. Método: estudio mixto, que integró 48 participantes de Oporto, Portugal, entre septiembre de 2018 y julio de 2019. Se utilizó un formulario para evaluar enfermedades y variables relacionadas con la salud, y una entrevista semiestructurada centrada en el automanejo de la enfermedad crónica. Los datos cualitativos se sometieron al análisis de contenido temático. Resultados: El 93,7\% de los participantes presentan comorbilidades, especialmente enfermedades cardiovasculares y endocrinas. De las entrevistas surgieron tres temas: Dominio de la gestión de medicamentos; Dificultad para comportarse siempre de forma saludable; Importancia de una relación cercana y de confianza con los profesionales de la salud. Conclusión: se evidenciaron las necesidades de autogestión de los ancianos, las cuales pueden ser minimizadas con intervenciones de enfermeras, destacando las áreas a intervenir y las estrategias a utilizar en programas orientados a promover su alfabetización en salud.

DESCRIPTORES: Ancianos; Automanejo; Enfermedad crónica; Envejecimiento; Enfermería.

Recebido em: 29/09/2021

Aprovado em: 06/10/2021

Editora associada: Luciana Puchalski Kalinke

Autor Correspondente:

Celeste Bastos

Escola Superior de Enfermagem do Porto - Porto, Portugal

E-mail: cbastos@esenf.pt

Contribuição dos autores:

Contribuições substanciais para a concepção ou desenho do estudo; ou a aquisição, análise ou interpretação de dados do estudo - Bastos C, Santos C, Martins MM, Fernandes C, Lima L; Elaboração e revisão crítica do conteúdo intelectual do estudo - Bastos C, Santos C, Fernandes C, Lima L. Todos os autores aprovaram a versão final do texto.

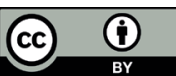

Copyright @ 2021 Este é um artigo em acesso aberto distribuído nos termos da Licença Creative Commons Atribuição, que permite o uso irrestrito, a distribuição e reprodução em qualquer meio desde que o artigo original seja devidamente citado. 